\title{
Assegurando o alinhamento estratégico da tecnologia de informação e comunicação: \\ o caso das unidades de refino da Petrobras*
}

\author{
Marcos Villas** \\ Marcus Fonseca*** \\ T. Diana L. v. A. de Macedo-Soares****
}

S UMÁRIO: 1. Introdução; 2. Referencial teórico; 3. Metodologia; 4. Resultados; 5. Discussão; 6. Conclusões.

Summary: 1. Introduction; 2. Conceptual framework; 3. Methodology; 4. Results; 5. Discussion; 6. Conclusions.

PALAVRAS-CHAVE: estratégia; planejamento estratégico; tecnologia de informação; alinhamento estratégico; balanced scorecard; setor de petróleo.

KEY WORDS: strategy; strategic planning; information technology; strategic fit; balanced scorecard; oil and gas sector.

A tecnologia de informação e comunicação (TIC) é fator crítico de competitividade das empresas. Embora alguns autores questionem o fato de a TIC poder ser considerada uma fonte de vantagem competitiva, há consenso quanto à necessidade do seu alinhamento à estratégia da empresa de modo que ela possa contribuir para a otimização do desempenho organizacional. Assegurar este alinhamento, ou seja, a consistência da estratégia da TIC com a da empresa, com vistas a aumentar sua efetividade, tornou-se particularmente importante com o acirramento da competição no setor de petróleo, em decorrência da abertura deste mercado no

* Artigo recebido em dez. 2004 e aceito em nov. 2005.

** MSc, doutorando do IAG/PUC-Rio. Professor de informática da PUC-Rio. Endereço: IAG/PUC-Rio — Rua Marquês de São Vicente, 225 — Gávea - CEP 22453-900, Rio de Janeiro, RJ, Brasil. E-mail: villas@inf.puc-rio.br.

*** MSc. Gerente de TI da Reduc/Petrobras. Endereço: Av. Maracanã, 1.302, ap. 401 — Tijuca - CEP 20511-001, Rio de Janeiro, RJ, Brasil. E-mail: marcusfonseca@petrobras.com.br.

**** PhD. Professora associada do IAG/PUC-Rio. Endereço: IAG/PUC-Rio — Rua Marquês de São Vicente, 225 — Gávea — CEP 22453-900, Rio de Janeiro, RJ, Brasil. E-mail: redes@strategyresearch.com. 
Brasil a partir de janeiro de 2002. Este artigo compartilha os resultados de um estudo de casos múltiplos nas unidades de negócios do refino da Petrobras e apresenta uma proposta de modelo conceitual para auxiliar a gestão da TIC no sentido de garantir o alinhamento de suas ações com as estratégias do negócio, com vistas a potencializar a contribuição da TIC para um melhor desempenho dessas unidades. Com base nos resultados da pesquisa, conclui-se que nelas existe um grau de alinhamento parcial da TIC à estratégia da empresa, sendo esta visão compartilhada pelos gerentes das suas diversas áreas de negócios. Como contribuição adicional, recomenda-se que a promoção do alinhamento estratégico da TIC seja construída no contexto do ciclo evolutivo da metodologia de avaliação do desempenho e gestão estratégica, balanced scorecard (BSC), que está sendo implementada na Petrobras.

Ensuring the strategic fit of information and communication technology: the case of Petrobras' oil refinery units

Information and communication technology (ICT) is a critical factor for company competitiveness. While some authors question the fact that ICT should be considered a source of competitive advantage, there is a strong consensus regarding the importance of ensuring its alignment with firm strategy so that it can contribute to the optimization of corporate performance. Ensuring this fit, that is, the consistency between the ICT strategy and the company's strategy, with a view to enhancing organizational effectiveness, became all the more important after the opening of Brazil's oil market in January 2002 and the ensuing increased competition in the oil sector in this country. The objectives of this article are to share the results of a multiple case study of the oil refinery units of Petrobras, and to propose a conceptual framework to help ICT management guarantee strategic fit between its actions and the units' business strategies, in order to optimize ICT's contribution to their performance. The study's results, in keeping with the perceptions of managers from different business areas at Petrobras, showed that there is merely a partial fit between ICT strategies and the business strategy of the oil refinery units at issue. As an additional contribution, the study suggests that the ICT strategic fit be promoted within the context of the evolutionary cycle of the "balanced scorecard" performance appraisal and the strategic management methodology that is being implemented throughout the company.

\section{Introdução}

\section{Alinhamento estratégico de TIC}

No atual ambiente de negócios, caracterizado pelo acirramento da concorrência e por mudanças crescentes, o potencial da tecnologia de informação e comunicação (TIC) está sendo utilizado para alavancar a vantagem competitiva de empresas na grande maioria das indústrias. Apoiadas pelo desenvolvimento das telecomunicações, as aplicações de TIC, com base principalmente na internet, redefiniram in- 
úmeros processos de negócios nas organizações (Davenport e Prusak, 1997; Luftman, 1996; Mata, Fuerst e Barney, 1995; McGee e Prusak, 1993).

De fato, neste novo ambiente mais competitivo, a utilização da TIC permite que novos atores e produtos substitutos tornem obsoletas fontes tradicionais de vantagens competitivas, como foi o caso da enciclopédia em CD-ROM Microsoft Encarta e sua ameaça aos negócios da Enciclopédia Britânica (Evans e Wurster, 1997).

Contudo, a obtenção de vantagens competitivas pelo uso da TIC implica um alinhamento estratégico, ou seja, uma coerência de propósitos entre as estratégias da TIC e as do negócio. Muitas empresas, principalmente as grandes, têm tido dificuldades em assegurar este alinhamento em todas as suas áreas e unidades.

O setor de petróleo no Brasil, depois da abertura, é um excelente exemplo desse ambiente novo mais competitivo no qual a TIC está tendo um papel estratégico e em que as empresas estão enfrentando este tipo de dificuldade. A seguir, apresentam-se algumas informações sobre este setor no Brasil pertinentes à investigação da questão do alinhamento estratégico da TIC na maior empresa do setor, a Petrobras.

\section{A Petrobras e sua estratégia de TIC}

As principais transformações que marcaram a história recente da indústria petrolífera brasileira foram: a quebra do monopólio estatal do petróleo exercido pela Petrobras em 1997; a instituição de novos agentes operadores e reguladores; e a introdução de um mercado competitivo no país por meio da liberação das importações de derivados e dos preços tabelados, e do livre acesso à infra-estrutura instalada pela Petrobras para distribuição dos seus produtos. Apesar desse novo cenário ter trazido muitos desafios, também foram criadas oportunidades para o mercado brasileiro de energia.

A partir de 1999, para fazer face às transformações do setor, a Petrobras tomou algumas iniciativas e fez investimentos em TIC para apoiar a efetiva implementação de sua nova estratégia em todas as suas unidades.

Contudo, no caso das suas unidades de negócios do refino, foco deste artigo, foram encontrados indícios relevantes da falta de alinhamento estratégico da TIC, tais como: investimentos realizados em TIC que não adicionavam valor à estratégia da organização; iniciativas de TIC implementadas sem que existisse um vínculo com alguma iniciativa estratégica; dificuldades na aprovação de orçamento para as iniciativas de TIC; duplicação de esforços no desenvolvimento de soluções de TIC.

Essas falhas comprometiam o potencial da utilização estratégica da TIC, que se tornou de fundamental importância para a Petrobras com a entrada em vigor da Lei do Petróleo (Brasil, 1997) iniciando o processo de desregulamentação do setor 
brasileiro de petróleo e favorecendo a entrada de novos atores na sua arena competitiva.

Alguns projetos já estavam em curso na Petrobras, especialmente nas unidades de negócios do refino, com o objetivo de melhor utilizar o potencial estratégico da TIC. Entre eles destacavam-se o desenvolvimento de uma arquitetura de informações do abastecimento, para orientar o desenvolvimento dos sistemas de informação em uso no abastecimento, vinculando-os às iniciativas estratégicas da companhia; a revisão da função TIC, visando a criação de um modelo único de governança de TIC na Petrobras.

A Petrobras também tinha adotado o balanced scorecard (BSC) para o acompanhamento do desdobramento de suas estratégias nas suas unidades de negócios. Esta metodologia, elaborada por Kaplan e Norton (1997), tenciona promover o alinhamento dos objetivos estratégicos com indicadores de desempenho, metas e planos de ação nos diferentes níveis e unidades da organização.

Apesar desses esforços, persistiam as dificuldades relacionadas ao alinhamento estratégico da TIC nas unidades de negócios do segmento de refino da empresa.

Assim, definiu-se como pergunta central à pesquisa como assegurar que a estratégia de TIC estivesse alinhada às estratégias empresariais das unidades de negócios do refino da Petrobras, de modo a contribuir efetivamente para um melhor desempenho da empresa nesse segmento?

Este artigo compartilha os resultados de uma investigação empírica conduzida nessas unidades para encontrar elementos de resposta a essa pergunta central da pesquisa, e propõe um modelo conceitual para auxiliar tomadores de decisão na avaliação e promoção contínuas do alinhamento estratégico da TIC.

Para isso, foi preciso responder às seguintes questões intermediárias:

t A estratégia de TIC é alinhada à estratégia de negócios das unidades de refino da Petrobras?

t As ações de TIC promovem de forma contínua o alinhamento estratégico?

t Quais são os fatores intervenientes, facilitadores e inibidores, do alinhamento estratégico da TIC nas unidades?

Com base na revisão da literatura conduzida em uma etapa preliminar da pesquisa, verificou-se que a questão do alinhamento estratégico da TIC, de modo a potencializar a utilização da TIC para obtenção de vantagens competitivas, tem sido foco de muitas pesquisas. Havia uma convergência entre os diversos estudos sobre o fato de que existia uma relação de mão dupla entre as estratégias de negócios e as opções de utilização da TIC, devendo o planejamento de TIC estar integrado ao planejamento estratégico da empresa. Ao mesmo tempo, essa revisão revelou que ainda havia algumas lacunas pertinentes à essa questão. 
Uma lacuna identificada referia-se aos fatores que dificultavam ou facilitavam a adequação estratégica da TIC. Uma exceção era a pesquisa realizada por Luftman (1996) que, mesmo assim, produzia resultados genéricos, não levando em consideração aspectos específicos de uma determinada organização, nem interações desta com o macroambiente. Outra lacuna verificada, dizia respeito à avaliação do desempenho do papel estratégico da TIC, geralmente limitada à avaliação dos investimentos em TIC (Moschetta, 1999).

A pesquisa em questão neste artigo buscou contribuir à superação destas lacunas. $\mathrm{O}$ artigo é dividido em seis partes. Nas duas a seguir, explicam-se o referencial teórico e a metodologia da pesquisa. Na quarta e quinta partes, apresentam-se e discutem-se seus principais resultados. Na última parte, formulam-se conclusões e propõese o modelo conceitual acima mencionado.

\section{Referencial teórico}

\section{Modelos para alinhamento estratégico da TIC}

Diversos modelos têm sido propostos com o objetivo de promover o alinhamento da estratégia de TIC com a da empresa. Brodbeck e Hoppen (2002), Keen (1991), Morton (1991), Henderson e Venkatraman (1993), Sauer e Yetton (1994) e Broadbent e Weill (1997) desenvolveram modelos cuja premissa era a utilização de abordagens estruturadas. Esses modelos relacionam o planejamento estratégico organizacional ao planejamento da TIC a fim de alcançar os objetivos organizacionais apoiados por tais tecnologias.

Os modelos conceituais que mais contribuíram para o desenvolvimento do referencial teórico desta pesquisa foram: o de Henderson e Venkatraman (1993), o de Brodbeck (2001), e o BSC de Kaplan e Norton (1997).

Henderson e Venkatraman (1993) elaboraram um modelo de alinhamento estratégico da TIC que tem como premissa que o planejamento da TIC deve ser dinâmico, de modo a contemplar a evolução dos ambientes interno e externo à organização, tanto de negócios quanto de TIC. Seu conceito de alinhamento estratégico é construído sobre dois grandes eixos: adequação estratégica (strategic fit) e integração funcional. O primeiro eixo, adequação estratégica, reflete a necessidade da estratégia considerar as relações entre os domínios internos e externos. No ambiente de negócios, o domínio externo refere-se à definição da estratégia empresarial, que é formulada em função do escopo do negócio, das decisões a respeito das competências necessárias para distinguir a organização da concorrência, e das decisões a respeito do modelo de governança. Já o domínio interno refere-se à infra-estrutura organiza- 
cional, definida em termos da estrutura administrativa, dos desenhos dos processos e das habilidades individuais. Para o ambiente de TIC, o modelo de alinhamento estratégico propõe que este seja analisado em termos do domínio externo, referindo-se à definição da estratégia da TIC, ou seja, como a organização está posicionada no mercado de TIC. Já o domínio interno é definido em termos da infra-estrutura da TIC, ou seja, como a infra-estrutura de TIC deve ser configurada e gerenciada, a partir das opções estratégicas da TIC. O segundo eixo, integração funcional, leva em consideração as implicações das escolhas feitas nos domínios de negócios que afetam o ambiente de TIC e vice-versa (integrações estratégica e operacional).

Os resultados da pesquisa de Henderson e Venkatraman levaram à definição de quatro perspectivas básicas de alinhamento, que são obtidas a partir da combinação das ações nos seguintes domínios:

t execução estratégica, onde a estratégia de negócios define a infra-estrutura organizacional, que, por sua vez, define os requisitos da infra-estrutura da TIC;

t potencial tecnológico, que implica o desenvolvimento de uma estratégia da TIC em resposta a uma estratégia de negócios, sendo a infra-estrutura da TIC elaborada a partir desses requerimentos;

t potencial competitivo, onde as escolhas estratégicas da TIC definem a estratégia de negócios e em conseqüência sua infra-estrutura;

t nível de serviço, onde o alinhamento estratégico resulta da qualidade e quantidade de serviços de TIC prestados à organização.

Por sua vez, o modelo elaborado por Brodbeck (2001) tem como objetivo estruturar e operacionalizar a promoção do alinhamento estratégico entre os planos de negócio e os de TIC durante a etapa de implementação da estratégia. Ao modelo de Henderson e Venkatraman (1993) são incorporados conceitos associados à gestão integrada das organizações. É considerada a crescente utilização de ferramentas de TIC promotoras de alinhamento nos níveis interno e operacional, por meio dos sistemas de gestão integrada (ERP). Esses sistemas requerem o redesenho dos processos da organização, de modo a favorecer o alinhamento dos planos de negócio e a TIC.

Uma das principais contribuições de Brodbeck (2001) é a construção de um arcabouço para identificar a promoção do alinhamento não somente na formulação da estratégia, como é o caso do modelo de Henderson e Venkatraman, mas, também, na implementação do processo de planejamento. Para tanto, Brodbeck (2001) acrescentou ao modelo de Henderson e Venkatraman alguns conceitos teóricos como: os estágios de integração entre os itens de negócio e TIC para o longo prazo (Teo, 1994); a etapa de implantação do processo de planejamento; e a instrumen- 
tação da gestão do processo de planejamento ao longo de seu horizonte (Kaplan e Norton, 1997).

Embora não seja sua proposta original, nesta pesquisa acredita-se que o modelo BSC pode ajudar na integração das atividades necessárias para assegurar o alinhamento estratégico da TIC às atividades de operacionalização da estratégia de negócios preconizadas nos modelos tanto de Henderson e Venkatraman (1993), quanto de Brodbeck (2001).

De fato, o BSC é fundamentalmente uma ferramenta que auxilia a implementação da estratégia ao ajudar a traduzi-la em objetivos específicos e mensuráveis. Sua utilização como um sistema de gestão estratégica fundamenta-se na sua capacidade de ajudar os executivos das organizações na comunicação das suas estratégias de negócio e no alinhamento estratégico das iniciativas operacionais e individuais. Como trata da definição das informações-chave para gerenciar o cumprimento da estratégia e dos processos gerenciais que promovem o alinhamento estratégico necessário para tal definição, a TIC possui um papel fundamental: o de guardiã e responsável pelo fluxo de informação na empresa que flui através de sistemas de informação. Assim, implica que a estratégia de TIC esteja alinhada aos objetivos organizacionais.

\section{Conceitos centrais}

Antes de apresentar as variáveis e os constructos utilizados na pesquisa, cabe deixar claro o que se entende por TIC. Inspirando-se em Luftman (1996), TIC foi definida na pesquisa como um conjunto de equipamentos (computadores, dispositivos de armazenamento e de comunicação), de aplicações (sistemas), de serviços (help-desk, desenvolvimento de aplicações, metodologias) e de pessoas (analistas, programadores, gerentes) utilizado pelas organizações para tratar dados e informações. Esta definição converge com a dos domínios estratégia da TIC e infra-estrutura da TIC do modelo de Henderson e Venkatraman (1993).

Por sua vez, estratégia foi definida de acordo com Macedo-Soares (2000) como propósito unificador que traz coerência e direção às ações e decisões de uma organização, especialmente no que diz respeito à alavancagem e à alocação de recursos necessários à melhoria ou à sustentação de seu desempenho, de acordo com a sua visão e considerando suas condições de ambientes interno e externo.

Dois outros conceitos inter-relacionados, centrais à pesquisa, são os de alinhamento e adequação estratégica. Segundo Henderson e Venkatraman (1993) o conceito de alinhamento estratégico é construído sobre dois grandes eixos: adequação estratégica (strategic fit) e integração funcional.

Conforme Hofer e Schendel (1979), há adequação quando há congruência entre todos os fatores estrategicamente significativos — os organizacionais, os macroam- 
bientais, a estratégia e o desempenho. Este conceito está explicitado em Barney (1996) e Macedo-Soares (2000), onde é destacado que, para ser eficaz, a estratégia deve ser congruente tanto com o ambiente externo à organização quanto com seus recursos, competências, objetivos e valores.

A integração funcional foi definida como o relacionamento entre as diferentes funções do negócio permitindo o fluxo da operação de maneira integrada, seja através da infra-estrutura organizacional, seja por meio da infra-estrutura da TIC (Luftman, 1996).

A esse propósito, cabe mencionar que na introdução a uma pesquisa empírica relativa à aplicação do modelo de Henderson e Venkatraman (1993), Avison e outros (2004) apresentam várias linhas de pensamento e definições relacionadas ao alinhamento estratégico da TIC, observando, no entanto, que todas elas dizem respeito à integração entre as estratégias de negócios e de TIC.

Nesta pesquisa adota-se, portanto, a definição de Henderson e Venkatraman (1993) para alinhamento estratégico, por abranger integração funcional e adequação estratégica.

\section{Variáveis e indicadores da pesquisa}

Com relação às variáveis e aos indicadores necessários para a avaliação do grau de alinhamento estratégico na dimensão etapa de formulação da estratégia da TIC, primeiro, foram usados os trabalhos de Henderson e Venkatraman (1993) para levantar indicadores pertinentes aos fatores que caracterizam as perspectivas de alinhamento - a variável denominada caracterização do alinhamento. Em seguida, Brodbeck (2001) e Luftman (1996) foram a base para levantar indicadores pertinentes à promoção do alinhamento — variável promoção do alinhamento.

Para verificar o grau de alinhamento estratégico na dimensão etapa de implementação da estratégia da TIC, foram utilizados elementos identificados no estudo de Brodbeck (2001) para definição de quatro variáveis: metodologia, gerenciamento, comprometimento e processos. Na dimensão consistência da TIC com o BSC, foram selecionados indicadores para avaliar o grau de suporte dado pela atividade de TIC aos objetivos estratégicos expressos no mapa estratégico referente ao desdobramento do BSC da Petrobras para o segmento de refino.

Por sua vez, para identificar os elementos genéricos que compõem as estruturas dos planos de negócio (missão, estratégias, objetivos, FCS, metodologia, participação e plano de ação) e da TIC (infra-estrutura de suporte, adequação e integração de processos e pessoas, consistência dos sistemas de informação) foram utilizados os elementos de planejamento definidos no estudo de Brodbeck (2001). 
No modelo de Henderson e Venkatraman (1993), estas mesmas variáveis são consideradas necessárias para caracterizar as perspectivas de alinhamento, definindo o escopo do negócio e o modelo de governança tanto na dimensão estratégia empresarial quanto na dimensão estratégia da TIC.

Os elementos do contexto organizacional, conforme afirma Brodbeck (2001), não são visíveis em seu modelo; contudo dizem respeito ao ambiente favorável para a promoção do alinhamento. Eles são representados pela clara definição e identificação do negócio, pela cultura organizacional e postura dos dirigentes, pela estrutura organizacional e pelo posicionamento da organização em face do ambiente externo (crescimento de mercado, competidores, inovação etc.).

Na tabela 1 são apresentadas as dimensões analisadas e suas variáveis, bem como os constructos e indicadores utilizados no estudo em questão neste artigo. Para alguns indicadores, foi adotada a classificação quanto ao grau de promoção de alinhamento proposta por Brodbeck (2001): A — alta promoção; P — promoção parcial; B - baixa promoção.

\section{Tabela 1}

Variáveis, constructos e indicadores do alinhamento estratégico

\begin{tabular}{|c|c|c|c|c|}
\hline Dimensão & Variável & Constructo & Indicador & $\begin{array}{l}\text { Questões no } \\
\text { questionário }\end{array}$ \\
\hline
\end{tabular}




\begin{tabular}{|c|c|c|c|c|}
\hline \multirow{10}{*}{$\begin{array}{l}\text { Etapa de } \\
\text { formulação da } \\
\text { estratégia da TIC }\end{array}$} & \multirow{5}{*}{$\begin{array}{l}\text { Caracterização do } \\
\text { alinhamento }\end{array}$} & $\begin{array}{l}\text { Liderança } \\
\text { executiva }\end{array}$ & $\begin{array}{l}\text { Líder } \\
\text { Visão da TIC } \\
\text { Visão do negócio } \\
\text { Priorizador }\end{array}$ & $\begin{array}{l}\text { A. } 1 \\
\text { A. } 4 \\
\text { A. } 7 \\
\text { A. } 10\end{array}$ \\
\hline & & Liderança de TIC & $\begin{array}{l}\text { Funcional } \\
\text { Técnica } \\
\text { Negócios } \\
\text { Serviços }\end{array}$ & $\begin{array}{l}\text { A. } 2 \\
\text { A. } 5 \\
\text { A. } 8 \\
\text { A. } 11\end{array}$ \\
\hline & & Foco da TIC & $\begin{array}{l}\text { Reativo } \\
\text { Proativo } \\
\text { Focado } \\
\text { Independente }\end{array}$ & - \\
\hline & & $\begin{array}{l}\text { Avaliação do } \\
\text { desempenho } \\
\text { da TIC }\end{array}$ & $\begin{array}{l}\text { Financeiro } \\
\text { Valor agregado } \\
\text { Produtos } \\
\text { Satisfação do } \\
\text { usuário }\end{array}$ & $\begin{array}{l}\text { A. } 3 \\
\text { A. } 6 \\
\text { A. } 9 \\
\text { A. } 12\end{array}$ \\
\hline & & $\begin{array}{l}\text { Método de } \\
\text { planejamento }\end{array}$ & $\begin{array}{l}\text { Focado em TIC } \\
\text { Focado em } \\
\text { negócios } \\
\text { Formalizado } \\
\text { ad-hoc }\end{array}$ & - \\
\hline & \multirow{5}{*}{$\begin{array}{l}\text { Promoção do } \\
\text { alinhamento }\end{array}$} & $\begin{array}{l}\text { Estratégias e } \\
\text { planos } \\
\text { da TIC bem } \\
\text { documentados e } \\
\text { explicitando } \\
\text { necessidade }\end{array}$ & $\mathrm{A} / \mathrm{P} / \mathrm{B}$ & B.1 \\
\hline & & $\begin{array}{l}\text { A alta } \\
\text { administração } \\
\text { participa do } \\
\text { planejamento da } \\
\text { TIC }\end{array}$ & $\mathrm{A} / \mathrm{P} / \mathrm{B}$ & $\begin{array}{l}\text { B. } 2 \\
\text { B. } 3\end{array}$ \\
\hline & & $\begin{array}{l}\text { O gerente de TIC } \\
\text { participa do } \\
\text { planejamento } \\
\text { estratégico }\end{array}$ & $\mathrm{A} / \mathrm{P} / \mathrm{B}$ & B.4 \\
\hline & & $\begin{array}{l}\text { Elevada visão do } \\
\text { relacionamento das } \\
\text { funções de TIC } \\
\text { com } \\
\text { a organização }\end{array}$ & A / P / B & $\begin{array}{l}\text { B. } 5 \\
\text { B. } 6 \\
\text { B. } 8 \\
\text { B. } 9\end{array}$ \\
\hline & & $\begin{array}{l}\text { Os recursos de TIC } \\
\text { são alocados e } \\
\text { priorizados a partir } \\
\text { do planejamento } \\
\text { estratégico }\end{array}$ & $\mathrm{A} / \mathrm{P} / \mathrm{B}$ & B.7 \\
\hline
\end{tabular}




\begin{tabular}{|c|c|c|c|c|}
\hline Dimensão & Variável & Constructo & Indicador & $\begin{array}{l}\text { Questões no } \\
\text { questionário }\end{array}$ \\
\hline \multirow{16}{*}{$\begin{array}{l}\text { Etapa de } \\
\text { implementação da } \\
\text { estratégia da TIC }\end{array}$} & \multirow{7}{*}{ Metodologia } & $\begin{array}{l}\text { Objetivos e } \\
\text { estratégias } \\
\text { organizacionais }\end{array}$ & $\mathrm{A} / \mathrm{P} / \mathrm{B}$ & C.1 \\
\hline & & $\begin{array}{l}\text { Envolvimento dos } \\
\text { gerentes e usuários }\end{array}$ & $\mathrm{A} / \mathrm{P} / \mathrm{B}$ & C. 2 \\
\hline & & Novos projetos & $\mathrm{A} / \mathrm{P} / \mathrm{B}$ & C.3 \\
\hline & & $\begin{array}{l}\text { Priorização de } \\
\text { projetos }\end{array}$ & $\mathrm{A} / \mathrm{P} / \mathrm{B}$ & C. 4 \\
\hline & & $\begin{array}{l}\text { Planejamento da } \\
\text { arquitetura da TIC }\end{array}$ & $\mathrm{A} / \mathrm{P} / \mathrm{B}$ & C.5 \\
\hline & & Avaliação & $\mathrm{A} / \mathrm{P} / \mathrm{B}$ & C.6 \\
\hline & & Melhores práticas & $\mathrm{A} / \mathrm{P} / \mathrm{B}$ & C.7 \\
\hline & \multirow{3}{*}{ Gerenciamento } & $\begin{array}{l}\text { Ferramentas de } \\
\text { TIC para execução } \\
\text { do planejamento }\end{array}$ & $\mathrm{A} / \mathrm{P} / \mathrm{B}$ & D.1 \\
\hline & & $\begin{array}{l}\text { Freqüência de } \\
\text { revisões e ajustes }\end{array}$ & $\mathrm{A} / \mathrm{P} / \mathrm{B}$ & D. 2 \\
\hline & & $\begin{array}{l}\text { Follow-up das } \\
\text { revisões do plano }\end{array}$ & $\mathrm{A} / \mathrm{P} / \mathrm{B}$ & D.3 \\
\hline & \multirow{4}{*}{ Comprometimento } & $\begin{array}{l}\text { Atendimento aos } \\
\text { objetivos }\end{array}$ & $\mathrm{A} / \mathrm{P} / \mathrm{B}$ & E.1 \\
\hline & & Motivação & $\mathrm{A} / \mathrm{P} / \mathrm{B}$ & E.2 \\
\hline & & $\begin{array}{l}\text { Envolvimento } \\
\text { gerencial }\end{array}$ & $\mathrm{A} / \mathrm{P} / \mathrm{B}$ & E.3 \\
\hline & & $\begin{array}{l}\text { Patrocínio da área } \\
\text { de negócios }\end{array}$ & $\mathrm{A} / \mathrm{P} / \mathrm{B}$ & E.4 \\
\hline & \multirow[t]{2}{*}{ Processos } & $\begin{array}{l}\text { Identificação de } \\
\text { oportunidades de } \\
\text { melhorias no } \\
\text { processo }\end{array}$ & $\mathrm{A} / \mathrm{P} / \mathrm{B}$ & F.1 \\
\hline & & $\begin{array}{l}\text { Monitoramento das } \\
\text { necessidades } \\
\text { internas }\end{array}$ & $\mathrm{A} / \mathrm{P} / \mathrm{B}$ & F.2 \\
\hline
\end{tabular}




\begin{tabular}{|c|c|c|c|c|}
\hline \multirow{4}{*}{$\begin{array}{l}\text { Consistência da } \\
\text { TIC com o BSC }\end{array}$} & \multirow{4}{*}{ Consistência } & $\begin{array}{l}\text { Suporte aos } \\
\text { objetivos da } \\
\text { perspectiva } \\
\text { financeira }\end{array}$ & $\mathrm{A} / \mathrm{P} / \mathrm{B}$ & G.1 \\
\hline & & \begin{tabular}{|l} 
Suporte aos \\
objetivos da \\
perspectiva \\
mercado \\
\end{tabular} & $\mathrm{A} / \mathrm{P} / \mathrm{B}$ & $\begin{array}{l}\text { G.2 } \\
\text { G.3 }\end{array}$ \\
\hline & & $\begin{array}{l}\text { Suporte aos } \\
\text { objetivos da } \\
\text { perspectiva } \\
\text { processos internos }\end{array}$ & A / P / B & $\begin{array}{l}\text { G.4 } \\
\text { G.5 } \\
\text { G.6 } \\
\text { G.7 }\end{array}$ \\
\hline & & $\begin{array}{l}\text { Suporte aos } \\
\text { objetivos da } \\
\text { perspectiva } \\
\text { crescimento de } \\
\text { aprendizado }\end{array}$ & A / P / B & G.8 \\
\hline
\end{tabular}

A metodologia da pesquisa é descrita sucintamente a seguir.

\section{Metodologia}

O principal método adotado na pesquisa foi um estudo de casos múltiplos da TIC nas unidades de refino do sistema Petrobras. A adoção deste método justificava-se com base nas recomendações de Yin (1994): tratava-se de uma pesquisa empírica sobre um fenômeno contemporâneo que precisava ser investigado no seu contexto real e cuja pergunta central era do tipo "como".

Participaram da pesquisa 35 executivos, entre os quais todos os gerentesgerais de TIC e de planejamento estratégico de cada uma das refinarias - Repar, RPBC, Revap, Replan, Recap, Reduc, Regap, RLAM, Lubnor e Reman; o gerente de TIC do abastecimento sede; e os gerentes de relacionamento da TIC Corporativa.

\section{Coleta e tratamento dos dados}

De acordo com Eisenhardt (1989) e Yin (1994), para assegurar a consistência dos resultados, foi adotada a estratégia de triangulação de métodos, levantando dados de várias fontes por diferentes meios: investigação documental, levantamento de percepções (tipo survey), entrevistas e observação participante por parte de um dos autores.

A pesquisa documental baseou-se em documentos disponibilizados pela empresa, pertinentes ao planejamento estratégico, plano de negócios do downstream, 
plano de negócio da unidade, planejamento estratégico de TIC e arquitetura de aplicativos de negócio do abastecimento.

O levantamento (survey) de percepções dos executivos de TIC e do negócio, a respeito do alinhamento estratégico da TIC, teve como principal instrumento um questionário predominantemente estruturado. Este questionário foi composto de 47 questões fechadas, em escala do tipo Likert de 5 graus, de modo a obter do respondente sua percepção sobre variáveis que traduzissem o grau de alinhamento estratégico. Seu teste piloto foi conduzido com cinco integrantes da equipe de TIC do abastecimento, cujas opiniões a respeito da clareza do questionário auxiliaram na elaboração do instrumento final.

Dos 35 questionários enviados (20 gerentes ligados ao negócio e 15 gerentes de TIC), foram obtidas 30 respostas, ou seja, uma taxa de retorno de 85,71\%. Com base na fórmula de Rea e Parker (2000), para definir amostras ou taxas de resposta no caso de pequenas populações, esta taxa foi considerada suficiente para efeito de generalização dos resultados a todos os executivos pertinentes à problemática sob estudo. Foi admitida também a hipótese simplificadora de que a população, em termos de percepção, era homogênea.

Conduziram-se também algumas entrevistas a fim de obter percepções mais aprofundadas a respeito de pontos mais complexos pertinentes ao problema central da pesquisa. Entre os entrevistados destacavam-se o gerente de tecnologia da informação do abastecimento, o gerente de engenharia da UN-Reduc e o gerente de tecnologia da informação da UN-Reduc.

Os dados levantados pela aplicação do questionário predominantemente estruturado foram tratados por meio de técnicas estatísticas descritivas simples (distribuição de freqüência). Foi verificado através de diferenças de médias se existia variação entre as percepções dos gerentes da organização e os gerentes de TIC, quanto ao alinhamento estratégico. Os depoimentos dos entrevistados foram tratados com técnicas de análise de conteúdo (Morse, 1994), para entender melhor as estratégias organizacionais e de TIC.

Interpretaram-se os dados por meio do seu confronto com os constructos e indicadores pertinentes, destacados no referencial teórico, à luz das limitações dos métodos utilizados. Dessa forma buscou-se uma explanação geral, gerando recomendações pertinentes à promoção do efetivo alinhamento da TIC, a partir da identificação dos fatores que inibem ou facilitam o alinhamento no escopo da unidade de análise da pesquisa. A utilização do BSC como um sistema de gerenciamento serviu de subsídio para a proposição de um modelo de alinhamento estratégico, onde as etapas necessárias à sua promoção estariam associadas às etapas do ciclo evolutivo do BSC. Também foram frutos dessa análise sugestões para a definição de indicadores que poderiam medir o alinhamento estratégico das diferentes 
unidades de negócio, baseados nos constructos identificados na teoria, contribuindo para a composição do BSC da companhia.

\section{Limitações metodológicas}

Ao adotar a estratégia de triangulação de métodos (Eisenhardt, 1989; Yin, 1994), confrontando dados de diferentes fontes e meios, foi possível minimizar em grande parte as limitações inerentes a cada um dos métodos utilizados.

Existia, obviamente, a limitação de todo estudo de caso de não permitir a generalização estatística dos seus resultados às demais unidades e empresas do setor. Essa não foi considerada relevante, pois o principal objetivo do estudo era gerar subsídios para uma generalização analítica e não-estatística, para poder ilustrar importantes elementos da teoria do alinhamento estratégico da TIC.

\section{Resultados}

Aqui são apresentados os resultados relativos à estratégia corporativa e às dimensões “etapa de formulação da estratégia da TIC”, "etapa de implementação da estratégia da TIC” e “consistência da TIC com o BSC”.

\section{Estratégia corporativa}

De acordo com os depoimentos dos entrevistados e as observações do pesquisador “participante”, a estratégia corporativa, definida em 2004, era a principal referência para a atuação da Petrobras. Direcionava suas principais ações, tendo como premissas a rentabilidade e a responsabilidade social, e visava a liderança no mercado brasileiro de petróleo e derivados, a consolidação como empresa de energia e a expansão seletiva no mercado internacional. Estas premissas, bem como a ênfase na internacionalização, estavam explícitas nas declarações da sua visão e missão (Petrobras, 2004):

\footnotetext{
A Petrobras será uma empresa integrada de energia com forte presença internacional e líder na América Latina, atuando com foco na rentabilidade e na responsabilidade social e ambiental.

Atuar de forma segura e rentável, com responsabilidade social e ambiental, nas atividades da indústria de óleo, gás e energia, nos mercados nacional e interna-
} 
Assegurando o Alinhamento Estratégico da Tecnologia de Informação e Comunicação

cional, fornecendo produtos e serviços adequados às necessidades dos seus clientes e contribuindo para o desenvolvimento do Brasil e dos países onde atua.

\section{Etapa de formulação da estratégia da TIC}

Com relação à variável caracterização do alinhamento da TIC, os dados dos levantamentos de percepção dos executivos de TIC e das unidades de negócios sugeriram fortemente que a perspectiva dominante deste alinhamento nas unidades de refino da Petrobras era a de execução estratégica, conforme o modelo de Henderson e Venkatraman (1993). Ou seja, os requisitos da infra-estrutura da TIC decorriam dos requisitos da infra-estrutura organizacional, que por sua vez eram definidos pela estratégia de negócios. A estratégia da TIC não existia ou não era relevante, pois não era ela que definia os requisitos de infra-estrutura da TIC.

\begin{tabular}{|c|c|c|c|c|c|}
\hline \multicolumn{6}{|c|}{$\begin{array}{l}\text { Tabela } 2 \\
\text { Estatísticas descritivas da seção A do questionário }\end{array}$} \\
\hline A - Perspectiva de alinhamento & Questão & Média & Desvio & $\begin{array}{l}\text { Perspectiva de } \\
\text { alinhamento }\end{array}$ & Somatório \\
\hline $\begin{array}{l}\text { O gerente da unidade } \\
\text { organizacional formula } \\
\text { estratégias a partir das } \\
\text { características/opções do } \\
\text { negócio }\end{array}$ & A. 1 & 3,9 & 1,0 & \multirow{3}{*}{$\begin{array}{l}\text { Execução } \\
\text { estratégica }\end{array}$} & \multirow{3}{*}{314} \\
\hline $\begin{array}{l}\text { O gerente de TIC da unidade } \\
\text { organizacional projeta e } \\
\text { implementa soluções de TIC } \\
\text { adequadas à estratégia de } \\
\text { negócios }\end{array}$ & A. 2 & 3,7 & 1,0 & & \\
\hline $\begin{array}{l}\text { O desempenho da TIC é avaliado } \\
\text { segundo critérios financeiros. A } \\
\text { TIC é analisada como um centro } \\
\text { de custos }\end{array}$ & A. 3 & 2,9 & 1,0 & & \\
\hline
\end{tabular}




\begin{tabular}{|c|c|c|c|c|c|}
\hline $\begin{array}{l}\text { O gerente da unidade } \\
\text { organizacional vislumbra } \\
\text { soluções de TIC para o negócio }\end{array}$ & A. 4 & 3,3 & 1,0 & \multirow{3}{*}{$\begin{array}{l}\text { Potencial } \\
\text { tecnológico }\end{array}$} & \multirow{3}{*}{292} \\
\hline $\begin{array}{l}\text { O gerente de TIC da unidade } \\
\text { organizacional consegue traduzir } \\
\text { as soluções vislumbradas pela } \\
\text { organização em ações práticas }\end{array}$ & A. 5 & 3,6 & 0,8 & & \\
\hline $\begin{array}{l}\text { A TIC é avaliada segundo sua } \\
\text { liderança tecnológica comparada } \\
\text { às outras unidades de negócios }\end{array}$ & A. 6 & 2,9 & 1,1 & & \\
\hline $\begin{array}{l}\text { O gerente da unidade } \\
\text { organizacional vislumbra novas } \\
\text { oportunidades de negócio por } \\
\text { meio da utilização da TIC }\end{array}$ & A. 7 & 3,0 & 1,0 & \multirow{3}{*}{$\begin{array}{l}\text { Potencial } \\
\text { competitivo }\end{array}$} & \multirow{3}{*}{307} \\
\hline $\begin{array}{l}\text { O gerente de TIC identifica } \\
\text { tendências de mercado que } \\
\text { possam auxiliar a organização }\end{array}$ & A. 8 & 3,6 & 0,9 & & \\
\hline $\begin{array}{l}\text { A TIC é avaliada pela qualidade } \\
\text { de suas soluções }\end{array}$ & A. 9 & 3,7 & 0,8 & & \\
\hline $\begin{array}{l}\text { O gerente da unidade } \\
\text { organizacional prioriza a } \\
\text { alocação de recursos } \\
\text { para a TIC }\end{array}$ & A.10 & 3,0 & 1,1 & \multirow{3}{*}{$\begin{array}{l}\text { Nível de } \\
\text { serviço }\end{array}$} & \multirow{3}{*}{288} \\
\hline $\begin{array}{l}\text { O gerente de TIC desenvolve } \\
\text { uma estratégia de TIC } \\
\text { independente da estratégia de } \\
\text { negócios, buscando um nível de } \\
\text { excelência em seus serviços }\end{array}$ & A.11 & 2,7 & 0,8 & & \\
\hline $\begin{array}{l}\text { A TIC é avaliada de acordo com } \\
\text { o nível de satisfação dos seus } \\
\text { usuários }\end{array}$ & A.12 & 3,9 & 0,8 & & \\
\hline
\end{tabular}

De fato, pela estatística descritiva, aplicada às respostas do questionário, verificou-se um maior nível de concordância entre os respondentes, tanto com relação ao papel da liderança executiva (questão A.1 = 3,9 > A.4, A.7 e A.10) quanto ao papel da liderança de TIC (A.2 = 3,7 > A.5, A.8 e A.11), no sentido de que ambos os constructos possuíssem os atributos necessários para a caracterização da perspectiva de alinhamento da TIC na organização com a de execução estratégica. Esta perspectiva de alinhamento dominante também pode ser observada pelo maior somatório de notas atribuídas ao bloco de questões A.1 + A.2 + A.3 (314). Esta percepção é reforçada pela caracterização do foco da TIC (tabela 1, terceiro constructo) como "reativo" 
(a área de TIC reage às necessidades de negócios quando estas são apresentadas) pois, segundo a análise documental realizada, a arquitetura que orienta a construção dos aplicativos de TIC na Petrobras tem, entre outros objetivos, o de aumentar o grau de atendimento aos requerimentos do negócio. Entretanto, não havia concordância que o desempenho da TIC (A.3, A.6, A.9 e A.12) fosse medido de acordo com a perspectiva de execução estratégica. Ao contrário, havia uma maior concordância que a TIC fosse avaliada de acordo com o nível de satisfação de seus clientes, característica necessária à classificação do alinhamento de acordo com a perspectiva nível de serviço.

\begin{tabular}{|c|c|c|c|c|}
\hline \multicolumn{5}{|c|}{$\begin{array}{l}\text { Tabela } 3 \\
\text { Estatísticas descritivas da seção B do questionário }\end{array}$} \\
\hline B - Promoção do alinhamento & Questão & Média & Desvio & Alinhamento \\
\hline Documentação de estratégias e planos de ação & B.1 & 3,1 & 0,8 & Parcial \\
\hline Plano de negócios situa necessidades de TIC & B. 2 & 3,4 & 0,7 & Parcial \\
\hline $\begin{array}{l}\text { Participação de gerentes de linha e de topo no } \\
\text { planejamento de TIC }\end{array}$ & B. 3 & 2,6 & 0,8 & Baixo \\
\hline $\begin{array}{l}\text { Participação do gerente de TIC no } \\
\text { planejamento estratégico da organização }\end{array}$ & B. 4 & 3,0 & 1,1 & Parcial \\
\hline $\begin{array}{l}\text { Compreensão dos objetivos organizacionais } \\
\text { pelo gerente de TIC }\end{array}$ & B.5 & 3,9 & 1,0 & Parcial \\
\hline $\begin{array}{l}\text { Adaptabilidade da TIC às mudanças } \\
\text { estratégicas } \\
\text { da organização }\end{array}$ & B.6 & 3,2 & 1,0 & Parcial \\
\hline $\begin{array}{l}\text { Alocação e priorização de recursos de TIC } \\
\text { a partir do planejamento estratégico }\end{array}$ & B.7 & 3,4 & 0,9 & Parcial \\
\hline $\begin{array}{l}\text { Educação da gerência de topo para a } \\
\text { importância estratégica de TIC }\end{array}$ & B. 8 & 2,9 & 0,9 & Baixo \\
\hline $\begin{array}{l}\text { Avaliação da importância estratégica das } \\
\text { tecnologias emergentes de TIC }\end{array}$ & B.9 & 3,0 & 1,0 & Parcial \\
\hline
\end{tabular}

Para caracterizar o grau de alinhamento (questões B.1 a G.8) conforme a tipologia especificada no estudo de Brodbeck (2001), considerou-se a seguinte correspondência deste com as médias observadas: "alto alinhamento” para médias iguais ou acima de 4, "alinhamento parcial” para médias iguais ou acima de 3 e abaixo de 4 e “baixo alinhamento” para médias abaixo de 3.

Com relação à variável promoção do alinhamento da TIC, os dados sugeriam que existia um grau de alinhamento parcial com relação à qualidade da documen- 
tação das estratégias e dos planos de ação de TIC (B.1 = 3,1). Segundo a análise documental não existia nenhum plano estratégico de tecnologia da informação formalizado na organização e nem documentação referente ao desdobramento claro dos objetivos estratégicos da organização, estabelecidos no BSC, em ações de TIC. Isso sugere um baixo grau de alinhamento com relação à qualidade da documentação das estratégias e dos planos de ação de TIC. O plano de negócios da organização expresso no documento Plano de Negócios do Downstream (PND) não faz referências explícitas a iniciativas de TIC, apresentando indícios de um grau parcial de promoção de alinhamento, pela baixa participação da alta administração da companhia em planejamento de TIC, fato corroborado pela percepção dos entrevistados (B.2 = 3,4).

A participação dos gerentes de linha e de topo nas atividades de planejamento de TIC demonstra conhecimento e discussão constante dos itens planejados, apesar do grau baixo de alinhamento observado $(B .3=2,6)$. A metodologia de planejamento estratégico da organização e principalmente a utilização do BSC estimulam a participação dos gerentes da organização, inclusive os gerentes de TIC, no processo de planejamento de negócios (B.4 = 3,0), mas apesar disto não há alta compreensão dos objetivos organizacionais por estes gerentes de TIC (B.5 = 3,9). A velocidade com a qual as adaptações em TIC são realizadas apontara para um grau parcial de alinhamento (B.6 = 3,2).

Para a alocação de recursos financeiros para TIC, feita anualmente, as entrevistas revelaram que era necessário justificar os investimentos de acordo com objetivos estratégicos. Por outro lado, permitia-se que "realocações" desses recursos fossem realizadas durante o exercício para outros projetos. No questionário, a alocação e a priorização de recursos de TIC a partir do planejamento estratégico foram consideradas tendo um grau parcial $(B .7=3,4)$ de alinhamento.

A investigação documental corroborou a percepção de baixo grau de alinhamento quanto à educação da gerência de topo para a importância estratégica de TIC (B.8 = 2,9). A falta de um processo que avalie a importância estratégica das soluções de TIC foi apontada nas entrevistas como o principal fator para o grau de alinhamento parcial deste item $($ B.9 $=3,0)$.

\section{Etapa de implementação da estratégia de TIC}

Na dimensão etapa de implementação da estratégia de TIC, a partir dos indicadores relacionados às variáveis metodologia, gerenciamento, comprometimento e proces- 
sos, os dados sugeriram que havia apenas um grau de promoção de alinhamento parcial.

\begin{tabular}{|c|c|c|c|c|}
\hline \multicolumn{5}{|c|}{$\begin{array}{l}\text { Tabela } 4 \\
\text { Estatísticas descritivas da seção C do questionário }\end{array}$} \\
\hline C - Método de implantação & Questão & Média & Desvio & Alinhamento \\
\hline $\begin{array}{l}\text { Considera os objetivos e estratégias } \\
\text { da organização }\end{array}$ & C. 1 & 3,8 & 0,6 & Parcial \\
\hline $\begin{array}{l}\text { Gerentes da organização envolvidos } \\
\text { nas decisões }\end{array}$ & C. 2 & 3,1 & 0,8 & Parcial \\
\hline $\begin{array}{l}\text { TIC identifica proativamente projetos de } \\
\text { suporte } \\
\text { aos objetivos estratégicos }\end{array}$ & C. 3 & 3,2 & 0,8 & Parcial \\
\hline $\begin{array}{l}\text { A priorização dos projetos é feita de acordo } \\
\text { com os objetivos estratégicos }\end{array}$ & C. 4 & 3,7 & 0,9 & Parcial \\
\hline $\begin{array}{l}\text { Existe um planejamento global de hardware } \\
\text { e software para a organização }\end{array}$ & C.5 & 3,5 & 1,0 & Parcial \\
\hline $\begin{array}{l}\text { Os resultados de TIC estão de acordo com } \\
\text { as expectativas da organização }\end{array}$ & C.6 & 3,4 & 0,9 & Parcial \\
\hline $\begin{array}{l}\text { A TIC implementa práticas recomendadas } \\
\text { por consultoria especializada }\end{array}$ & C.7 & 3,1 & 1,0 & Parcial \\
\hline
\end{tabular}

As respostas em questão indicaram um grau de promoção de alinhamento parcial no caso da variável metodologia (médias de 3,1 a 3,8 para as questões de C.1 a C.7). Também sugeriram que havia envolvimento parcial das gerências de topo no processo de implantação das soluções e pouca proatividade de TIC na identificação de novos projetos que suportem a estratégia da companhia. Embora fossem utilizadas “as melhores práticas do mercado” para TIC, de acordo com consultorias especializadas externas à empresa, segundo a percepção dos gestores da área de negócio das unidades pesquisadas, a TIC não implementava estas práticas.

Tabela 5

Estatísticas descritivas da seção D do questionário 


\begin{tabular}{lcccc}
\hline D — Gestão das ações de TIC & Questão & Média & Desvio & Alinhamento \\
\hline $\begin{array}{l}\text { Acompanhamento sistemático da eficácia das } \\
\text { ações implementadas }\end{array}$ & D.1 & 3,3 & 1,0 & Parcial \\
$\begin{array}{l}\text { Reuniões de acompanhamento da } \\
\text { implementação das ações de TIC }\end{array}$ & D.2 & 3,3 & 1,2 & Parcial \\
$\begin{array}{l}\text { A TIC possui indicadores de acompanhamento } \\
\text { das ações implementadas }\end{array}$ & D.3 & 3,6 & 1,0 & Parcial \\
\hline
\end{tabular}

De acordo com os indicadores definidos para caracterização da variável gerenciamento, os dados também apontaram para um grau parcial de promoção de alinhamento estratégico da TIC nesta etapa (D.1 = 3,3; D.2 = 3,3; D.3 = 3,6). Havia percepções diferentes entre os gestores da área de negócios e os gestores de TIC. Se, por um lado, havia diversos indicadores estabelecidos para medir a qualidade do serviço e o desempenho de alguns equipamen-tos (principalmente servidores e rede), por outro, não existiam indicadores que medissem a eficácia de uma determinada solução, em especial o retorno do investimento realizado.

\section{Tabela 6}

Estatísticas descritivas da seção E do questionário

E-Comprometimento Questão Média Desvio Alinhamento

Os empregados de TIC da organização

cumprem

as metas negociadas

E.1

3,7

0,7

Parcial

Os benefícios desses empregados estão

vinculados às metas negociadas

E.2

3,5

0,9

Parcial

Os gerentes da organização estão

comprometidos em auxiliar a TIC na

implantação de suas ações

E.3

3,2

0,9

Parcial

As ações de TIC são sempre patrocinadas por

alguma gerência da estrutura organizacional

E.4

3,8

0,8

Parcial

A variável comprometimento também apresentou grau parcial de promoção de alinhamento pois não houve consenso quanto ao cumprimento de metas negocia- 
das pelos funcionários de TIC (E.1 = 3,7), embora seus benefícios estivessem vinculados a estas metas $(\mathrm{E} .2=3,5)$. O patrocínio da gerência da organização é um fator crítico de sucesso para os projetos de TIC $($ E.3 = 3,2). Conforme foi observado anteriormente, nem sempre os projetos de TI estão vinculados diretamente a objetivos estratégicos. Neste caso, o patrocínio não é alcançado levando muitos projetos a fracassar $($ E.4 = 3,8).

Tabela 7

Estatísticas descritivas da seção F do questionário

\begin{tabular}{|c|c|c|c|c|}
\hline $\mathrm{F}$ - Processos & Questão & Média & Desvio & Alinhamento \\
\hline $\begin{array}{l}\text { A organização de TIC identifica } \\
\text { de melhorias nos processos de } n \\
\text { meio } \\
\text { de suas ações }\end{array}$ & F.1 & 3,5 & 0,7 & Parcial \\
\hline $\begin{array}{l}\text { A organização de TIC monitora } \\
\text { necessidades internas do negóci } \\
\text { capacidades de TIC } \\
\text { para atender estas necessidades }\end{array}$ & F. 2 & 3,3 & 0,7 & Parcial \\
\hline
\end{tabular}

A capacidade de TIC em promover a melhoria dos processos e identificar novos projetos estratégicos foi avaliada pelos entrevistados como tendo grau parcial de promoção de alinhamento (F.1 = 3,5 e F.2 = 3,3).

\section{Consistência da TIC com o BSC}

Na dimensão consistência da TIC com o BSC, os dados indicaram tanto um alto grau quanto um grau parcial de promoção de alinhamento da TIC.

Foi verificado um alto grau de promoção de alinhamento no caso das variáveis que mediram a consistência da TIC aos seguintes objetivos estratégicos: proteção do mercado $(G .2=4,1)$, melhorar o nível de serviço ao cliente final $(\mathrm{G} .3=4,5)$, aumentar a integração entre as áreas do downstream $(\mathrm{G} .4=4,3)$ e qualidade dos produtos (G.5 = 4,1). Já no caso dos objetivos estratégicos redução do custo de refino $(\mathrm{G} .1=3,9)$, informações de mercado (G.6 = 3,6), promoção de segurança, meio ambiente e saúde (G.7 = 3,7) e desenvolvimento de competências (G.8 = 3,9), foi verificado um grau apenas parcial de promoção de alinhamento para as variáveis que mediram a consistência da TIC com esses objetivos. 


\section{Tabela 8}

Estatísticas descritivas da seção G do questionário

G - Consistência entre as ações de TIC e os objetivos estratégicos da organização

Objetivo de redução de custos Questão Média Desvio Alinhamento

Objetivo de proteção do mercado

G. 1

3,9

1,0

Parcial

Objetivo de melhorar nível de serviço ao

G. 2

4,1

1,0

Alto

cliente final

G.3

4,5

0,8

Alto

Objetivo de integração entre as áreas do

G.4

4,3

0,9

Alto

downstream

Objetivo de qualidade de produtos

Objetivo de informações de mercado

G.5

4,1

1,0

Alto

Objetivo de promoção de SMS

G.6

3,6

1,1

Parcial

G.7

3,7

0,9

Parcial

Objetivo de desenvolvimento de competências estratégicas

G.8

3,9

1,0

Parcial

\section{Discussão}

Nesta seção discutem-se os resultados apresentados à luz do referencial teórico, visando responder às questões da pesquisa.

A estratégia da TIC é alinhada à estratégia de negócios das unidades de refino da Petrobras?

Os resultados do estudo de caso indicaram que, de uma forma geral, o contexto organizacional nas unidades de negócios do refino da Petrobras fornecia condições favoráveis à promoção do alinhamento estratégico da TIC. Entre os fatores destacados por Brodbeck (2001), apenas no caso da questão da proximidade entre a área de TIC e a área de negócios, os resultados indicaram um baixo grau na promoção do alinhamento. Note, contudo, que se identificou a falta de um planejamento que explicite as estratégias da TIC, bem como a ausência de um desdobramento formal dos objetivos estratégicos em ações de TIC. Este fato inibia a promoção do alinhamento, de acordo com a perspectiva do potencial tecnológico necessário para o pleno alinhamento estratégico da TIC. 
Embora os resultados do estudo relativos à consistência das ações de TIC confirmassem a adequação das ações de TIC à estratégia de negócios, na medida em que foi verificado um alto grau de alinhamento dos produtos e serviços de TIC aos objetivos de negócio, inferiu-se que este fato não advinha de uma estratégia da TIC decorrente formal e explicitamente da estratégia de negócios.

\section{As ações de TIC promovem de forma contínua o alinhamento estratégico?}

Conforme mencionado, o estudo revelou que faltava na organização um processo sistemático de desdobramento dos objetivos estratégicos em objetivos de TIC. Este fato corroborava para que não houvesse um efetivo desdobramento desses objetivos em ações individuais, dificultando a percepção quanto ao cumprimento das metas negociadas. Da mesma forma, os benefícios individuais não estavam associados diretamente ao atingimento das metas individuais, fazendo com que a prática de gestão do desempenho, adotada na organização, não alcançasse plenamente seus objetivos. Da mesma forma, não foram identificadas condições para as perspectivas potencial competitivo e nível de serviço (Henderson e Venkatraman, 1993), notadamente no que se referia à liderança e condução do processo estratégico por TIC. Concluiu-se assim que as ações de TIC promoviam apenas parcialmente o alinhamento estratégico.

Quais são os fatores intervenientes, facilitadores e inibidores, do alinhamento estratégico da TIC nas unidades?

Dos cinco principais fatores facilitadores do alinhamento estratégico da TIC propostos por Luftman, Papp e Brier (1999) apenas a compreensão do negócio pela TIC mostrou-se presente nas unidades de refino da Petrobras. Os fatores "patrocínio do executivo principal”, "envolvimento da TIC no desenvolvimento da estratégia”, "parceria entre a TIC e a área de negócio” e “projetos de TIC são priorizados de forma adequada” não foram identificados nessas unidades. Além destes, a pesquisa verificou que a utilização do BSC como um sistema de gerenciamento, a prática de acompanhamento de indicadores e a capacidade financeira da organização eram fatores que facilitavam a utilização estratégica da TIC.

Entretanto, também foram relacionados outros fatores que dificultavam o alinhamento estratégico da TIC na organização, tais como a falta de patrocínio e o baixo envolvimento da área de negócios no desenvolvimento da estratégia de TIC.

\section{Conclusões}


Acredita-se que esta pesquisa atingiu em grande parte seu objetivo ao identificar como assegurar que a estratégia da TIC estivesse alinhada às estratégias empresariais das unidades de negócios do segmento de refino da Petrobras, de modo a contribuir efetivamente para seu melhor desempenho. Indicou como perspectiva de alinhamento dominante no segmento de refino da Petrobras a execução estratégica do modelo de Henderson e Venkatraman (1993), segundo a qual a estratégia de negócios define a infra-estrutura organizacional, a qual, por sua vez, define os requisitos da infra-estrutura da TIC. Ao mesmo tempo, revelou a falta das outras perspectivas deste modelo, notadamente a de potencial tecnológico. Não foi surpresa, portanto, a baixa participação dos executivos de TIC na formulação das estratégias de negócios nas unidades de refino da Petrobras e a ausência de estratégia explícita de TIC.

O novo cenário competitivo no qual a Petrobras se insere, após a quebra do monopólio estatal em 2002, estabelece precondições para que se utilize a TIC de forma estratégica. A identificação dos fatores que inibem a promoção do alinhamento estratégico na organização permite que sejam elaborados planos de ação com o objetivo de criar condições para reduzir a sua influência negativa. Entre as ações mais importantes, destaca-se a necessidade de conscientizar a gerência da organização a respeito da importância estratégica da TIC e seu potencial tecnológico de auxiliar a organização a conquistar vantagem competitiva.

Uma contribuição adicional da pesquisa foi recomendar a promoção contínua do alinhamento estratégico da TIC dentro do ciclo do BSC. Esta proposta foi baseada na identificação de que muitas das variáveis presentes nos modelos de alinhamento estratégico são convergentes às ações estabelecidas no ciclo evolutivo do BSC, e no fato de que podem ser utilizadas as medidas do BSC para comunicar a estratégia e alinhar iniciativas individuais, organizacionais e departamentais, para alcançar um objetivo comum. Argumenta-se aqui que a utilização do BSC, conforme a definição de Kaplan e Norton (1997), estabelece condições para este aprendizado.

Esta pesquisa, como contribuição principal, propõe uma síntese entre os elementos necessários à promoção do alinhamento e os princípios gerenciais do BSC. Essa síntese é possível porque aos elementos do processo de tradução da visão no BSC em objetivos estratégicos precisos estão associados os elementos de formulação da estratégia (modelo de Henderson e Venkatraman, 1993). Da mesma forma, nas outras fases do ciclo do BSC, há elementos que se associam aos elementos de implantação da estratégia proposta por Brodbeck (2001). Sugere-se que os fatores críticos para o alinhamento em questão sejam avaliados de acordo com sua contribuição para cada etapa do ciclo evolutivo do BSC, utilizando-se o modelo apresentado na figura.

O modelo proposto explora o fato de que a Petrobras utiliza o BSC como um sistema de gerenciamento, o que facilita o alinhamento das etapas do planejamento 
estratégico da TIC. A sua utilização permitiria que as ações estratégicas de TIC tivessem a necessária estrutura para seu desdobramento sistemático a partir das estratégias do negócio, assegurando integração e articulação maior entre os objetivos de TIC e os do negócio.

Um dos resultados mais relevantes da pesquisa foi identificar condições para que seja definido um conjunto de indicadores de desempenho da TIC aderente ao acompanhamento dos seus objetivos estratégicos.

Modelo para assegurar alinhamento estratégico da TIC por meio do BSC

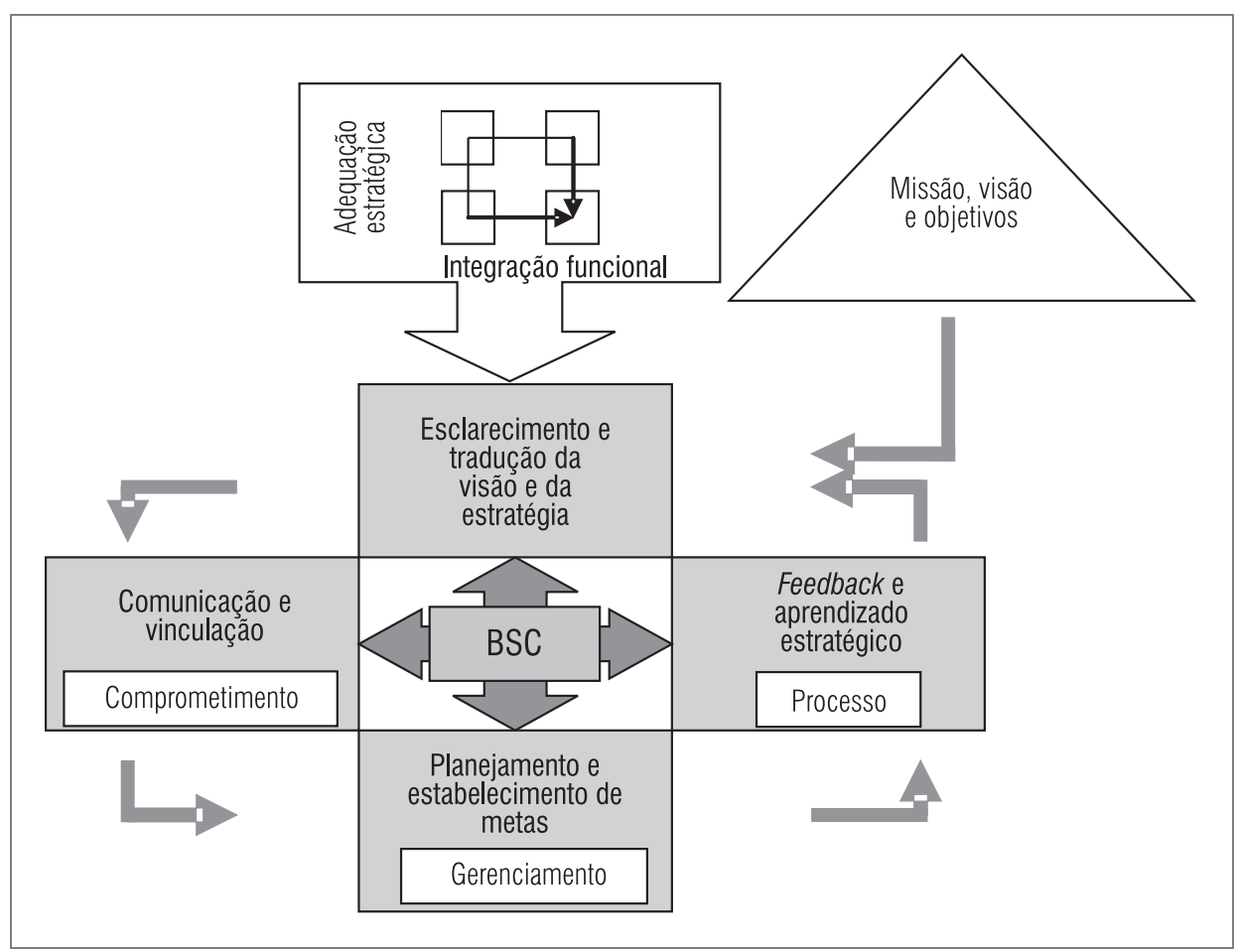

A falta de um conjunto consistente de indicadores foi observada quando da verificação do grau de alinhamento durante a fase da implementação das ações de TIC, de acordo com o modelo de Brodbeck (2001), onde foi ressaltado que existe a necessidade de um acompanhamento sistemático por parte da TIC das ações implantadas. Como sugestão, esta pesquisa enfatiza dar mais importância ao estabelecimen- 
to de indicadores por parte da TIC para monitorar, por exemplo, o número de reuniões de acompanhamento das ações de TIC e para verificar a eficácia dessas ações.

Apesar do escopo desta pesquisa estar limitado às unidades de negócios do segmento de refino da Petrobras, seus principais resultados podem ser aproveitados, principalmente por aquelas empresas que adotam o BSC como um sistema gerencial. Com auxílio do modelo apresentado na figura, elas poderiam utilizar a proposição desta pesquisa de promover o alinhamento estratégico dentro do ciclo evolutivo do BSC, para criar uma estratégia de TIC que permita explorar seu potencial de contribuir para a otimização do desempenho da empresa.

\section{Referências bibliográficas}

AVISON, D. et al. Using and validating the strategic alignment model. Journal of Strategic Information Systems, v. 13, n. 3, p. 223-246, 2004.

BARNEY, J. B. Gaining and sustaining competitive advantage. Reading, MA: Addison-Wesley, 1996.

BRASIL. Lei n. 9.478, de 6 de agosto de 1997. Dispõe sobre a política energética nacional, as atividades relativas ao monopólio do petróleo, institui o Conselho Nacional de Política Energética e a Agência Nacional do Petróleo e dá outras providências.

BROADBENT, M.; WEILL, P. Management by maxim: how business and IT managers can create IT infrastructures. Sloan Management Review, v. 38, n. 3, p. 77-92, Spring 1997.

BRODBECK, A. F. Alinhamento estratégico entre os planos de negócio e de tecnologia de informação: um modelo operacional para a implementação. 2001. 332 f. Tese (Doutorado em Administração) - Universidade Federal do Rio Grande do Sul, Porto Alegre, 2001.

; HOPPEN, N. Modelo de alinhamento estratégico para implementação dos planos de negócio e de tecnologia da informação. In: ENCONTRO ANUAL DA ANPAD, 25., Salvador, 2002. Anais... Salvador: Anpad 2002.

DAVENPORT, T.; PRUSAK, Laurence; Information ecology. New York: Oxford University Press, 1997.

EISENHARDT, K. M. Building theories from case study research. The Academy of Management Review, v. 14, n. 4, Oct. 1989.

EVANS, P. B.; WURSTER, T. Strategy and the new economics of information. Harvard Business Review, v. 75, n. 5, p. 71-82, Sept./Oct. 1997.

GIL, A. C. Como elaborar projetos de pesquisa. 3. ed. São Paulo: Atlas, 1991. 
GRANT, R. M. Contemporary strategy analysis. Malden, Mass.:Blackwell Business, 1998.

HENDERSON, J. C.; VENKATRAMAN, N. Strategic alignment: leveraging information technology for transforming organizations. IBM Systems Journal, v. 32, n. 1, p. 4-16, 1993.

HILL, C.; JONES, G. Strategic management theory — an integrated approach. Boston: Houghton Mifflin Company, 1998.

HOFER, C.; SCHENDEL, D. E. Strategy formulation. Boston: Little, Brown \& Co., 1979.

KAPLAN, R. S.; NORTON, D. P. A estratégia em ação. Rio de Janeiro: Campus, 1997.

KEEN, P. Every manager's guide to information technology. Boston: Harvard Business School Press, 1991.

LEDERER, A. L.; MENDELOW, A. L. Coordination of information system plans with business plans. Journal of Management Information Systems, Fall 1989.

LUFTMAN, J. Competing in the information age. Oxford University Press, 1996.

— PAPP, R.; BRIER, T. Enablers and inhibitors of business-IT alignment. Communications of AIS, v. 1, n. 11, Mar. 1999.

MACEDO-SOARES, T. D. L. v. A. An integrative model for strategic management analysis: application to organizations in Brazil. In: INFORMS-KORMS CONFERENCE. Proceedings... 2000, Seoul, p. 460-467.

MATA, F.; FUERST, W.; BARNEY, J. Information technology and sustained competitive advantage: a resource-based analysis. MIS Quarterly, p. 487-505, Dec. 1995.

MCFARLAN, F. W. Information technology changes the way you compete. Harvard Business Review, v. 62, n. 3, p. 98-103, May/June 1984.

MCGEE, J.; PRUSAK, L. Managing information strategically. [Hoboken]: John Wiley \& Sons, 1993.

MORSE, J. M. Critical issues in qualitative research methods. London: Sage, 1994.

MORTON, Michael S. Scott. The corporation of the 1990s: information technology and organizational transformation. New York: Oxford University Press, 1991.

MOSCHETTA, R. Alinhamento da tecnologia da informação com a estratégia empresarial: a percepção das maiores organizações gaúchas. 1999. Dissertação (Mestrado) — Universidade do Vale do Rio dos Sinos.

PETROBRAS. Plano estratégico Petrobras 2015. Maio 2004. Disponível em: <www2.petrobras. com.br/Petrobras/portugues/visao/vis_plano.htm>. Acesso em: 24 nov. 2004. 
PORTER, M. Estratégia competitiva, técnicas para análise de indústrias e da concorrência. 18. ed. Rio de Janeiro: Campus, 1986.

- Vantagem competitiva, criando e sustentando um desempenho superior. 14. ed. Rio de Janeiro: Campus, 1989.

REA, L. M.; PARKER, R. A. Metodologia de pesquisa — do planejamento à execução. São Paulo: Pioneira, 2000.

REICH, B. H. Investigating the linkage between business objectives and information technology objectives: a multiple case study in the insurance industry. 1992. $458 \mathrm{f}$. Tese (Doutorado) - University of British Columbia, Vancouver.

ROCKART, J. F. Chief executives define their own data needs. Harvard Business Review, p. 8193, Mar./Apr. 1979.

; MORTON, M. S. Implications of changes in information technology for corporate strategy. Interfaces, v. 14, n. 1, p. 84-95, 1984.

SAUER, C.; YETTON, P. The dynamics of fit and the fit of dynamics: aligning IT in a dynamic organization. In: ICIS. Proceedings... 1994, Vancouver, p. 41-50.

TEO, T. S. H. Integration between business planning and information system planning: an evolutionary-contingency perspective. 1994. PhD (Dissertation) — University of Pittsburgh.

VERGARA, S. C. Projetos e relatórios de pesquisa em administração. São Paulo: Atlas, 1997.

YIN, R. K. Case study research: design and methods. 2. ed. London: Sage, 1994. 